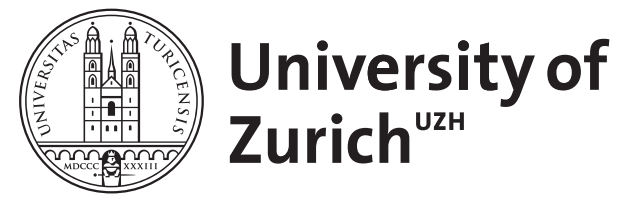

\title{
Rare appearance, rare location and unusual patient age
}

Pineker, Viktor ; Marques Maggio, Ewerton ; Huber, Alexander ; Pangalu, Athina ; Valavanis, Antonios ; Winklhofer, Sebastian

DOI: https://doi.org/10.1007/s00062-017-0582-z

Posted at the Zurich Open Repository and Archive, University of Zurich ZORA URL: https://doi.org/10.5167/uzh-136859

Journal Article

Accepted Version

Originally published at:

Pineker, Viktor; Marques Maggio, Ewerton; Huber, Alexander; Pangalu, Athina; Valavanis, Antonios; Winklhofer, Sebastian (2017). Rare appearance, rare location and unusual patient age. Clinical Neuroradiology, 27(4):521-524.

DOI: https://doi.org/10.1007/s00062-017-0582-z 


\title{
Title Page
}

Rare Appearance, rare Location, and unusual Patient Age: Report of an osteolytic Fibrous Dysplasia in the Temporal Bone of a 52-year-old Patient.

\author{
Word count: 1101
}

Type of manuscript: correspondence 


\section{Introduction}

Fibrous Dysplasia (FD) is a benign developmental disorder in which normal bone is replaced by conjunctive tissue and woven bone [1]. In the head and neck region, it may cause bone-dysplasia, obstruction of sinuses and the skull foramina, as well as bony instability, resulting in neural lesions, meningitis, or malignant transformation. It is the most common genesis of bone dysplasia in childhood and young patients [2].

There are three subtypes: First, the monostotic form (70\%), affecting a single bone. Second, the polyostotic form (25\%), also known as Morbus Jaffé-Lichtenstein, affecting multiple bones. Third, if the polyostotic form combines with dermal hyperpigmentation and endocrine dysfunction, it is classified as the rare McCune-Albright-Syndrome [1].

The following case represents an atypical manifestation of FD and shall increase awareness for this entity. 


\section{Case Report}

This case study was performed in accordance with the ethical standards laid down in the 1964 Declaration of Helsinki and its later amendments. The patient provided written informed consent.

\section{Patient presentation}

A 52-year-old, female patient presented in our emergency department with three days of persisting fluctuations of blood pressure and analgesic resistant headaches paired with nausea. The auricular system demonstrated no pathological symptoms. Subsequently, to rule out an intracranial pathology, a computed tomography (CT) as well as a magnetic resonance imaging (MRI) examination of the head was performed.

\section{Imaging}

CT demonstrated an osteolytic lesion with a well-defined margin in the left mastoid, measuring approximately $3 \mathrm{~cm}$ in cranio-caudal direction (Fig. 1). The cortical bone was markedly thinned, with suspicion of a short dehiscence adjacent to the posterior fossa. Therefore, a cortical breakthrough could not be excluded by imaging.

In MRI, the lesion appeared inhomogeneous hypo-, to isointense in T2-weighted (w) images and suggested a dorsal hemorrhagic component with relatively homogeneous uptake of contrast agent (Fig. 2). Besides that, mucus retention in parts of the lesion and fluid of individual mastoid cells was apparent. No adjacent soft tissue involvement was visible.

The initial imaging diagnosis was - even after thorough discussion with experienced head and neck radiologist - unclear with plasmocytoma, metastatic disease, ossifying fibroma, fibrosarcoma, and lymphoma in the list of differential diagnoses. The unknown dignity indicated a further histological analysis. Alternative possible strategies would have included a 
fine needle biopsy or a "wait-and see strategy", however, summarizing the individual aspects, the therapy of choice was surgery in this case.

Surgery and Histopathology

An explorative biopsy was performed with consequent resection of the tumor. Surgery revealed a solid and chondrotic tumor that was connected to the surrounding bone via small branches (Fig. 3). Furthermore cholesterol-like material was detected in a big mastoid cell that was occluded by tumor.

The initial frozen section analysis was consistent with FD, leaving the differential diagnosis of fibrous meningioma possible. The examination of formalin fixed paraffin embedded tissue yielded the diagnosis of a fibro-osseous lesion consistent with FD (Fig. 4). A confirmatory GNAS Mutation analysis followed and revealed a G-protein activating point mutation (p.R201C, c.601C>T). As expected smooth muscle actin (SMA) stained the spindle cells. MIB1 labeled heterogeneously the spindle cells (up to $10 \%$ in the periphery). Despite unusual patient age, localization, and imaging findings, the typical morphology and genetics confirmed the diagnosis of monostotic FD.

The patient recovered well after surgery without any new persisting symptoms. 


\section{Discussion}

The correct imaging diagnosis of cases with FD in the head and neck region might be challenging $[3,4]$. The disease is commonly diagnosed in children or young adults, with $75 \%$ of the patients less than 30 years of age [5]. A higher patient age, as it was the case in our patient, might hamper the diagnosis and might tend to favor other differential diagnoses, such as metastatic disease. Frequently, FD impacts the ribs (28\%), followed by the proximal femur $(23 \%)$ and the craniofacial skeleton $(10-25 \%)[6,7]$. Referring to patients with a manifestation in craniofacial bones, a series of 21 cases has been analyzed and concluded, that FD most commonly affects the ethmoid (71\%) and sphenoid bone (41\%) and only in $24 \%$ the temporal bone [8]. Mahajan et al. stated that the affection of craniofacial bones is very rare with the following order of involved bones: frontal $>$ sphenoid $>$ ethmoid $>$ maxillary $>$ mandible $>$ zygomatic $>$ occipital $>$ temporal [9].

The patients symptoms align with the location of the lesion, led by atypical facial pain, headache, nerve compression and sinus symptoms [6]. In case of a FD in the temporal bone, the most reported clinical findings are conductive hearing loss and obstruction of the external auditory canal [2]. A solitary manifestation of FD in the mastoid is rare and there is not much literature dealing with it $[2,10]$.

FD represents a benign entity, however there is a possibility for spontaneous malignant transformation in about $0.5 \%$, with two thirds representing osteosarcoma. Other possibilities of transformation are fibrosarcoma, chondrosarcoma, or malignant fibrohistiocytoma [11].

Usually, no invasive therapy is required. However, in cases of symptomatic patients, surgery might result in a reduction of symptoms, functional restoration, or aesthetic improvement [11]. 
The most common imaging feature of FD is an expansile lesion with a homogenous ground-glass appearance which is described in approximately 56\% of FD cases (Fig. 5) [3]. The surrounding cortical layer is frequently thinned. Other imaging patterns include a sclerotic appearance and an osteolytic or cystic manifestation, with the letter being rarely described [12]. CT is commonly the first-line evaluation tool in FD to assess osseous structures [11]. Imaging appearance in MRI might demonstrate a remarkable variability and might resemble a malignant finding. Nevertheless, MRI is suitable to evaluate the lesions form and size, as well as nearby neurovascular or temporal structures which can be relevant for the patient's symptoms and treatment. Typically, FD appears with intermediate to low signal intensity in $\mathrm{T} 1 \mathrm{w}$, intermediate to low signal in $\mathrm{T} 2 \mathrm{w}$, and a heterogenous gadolinium enhancement [12]. Mucus retention is frequently seen in FD of the midface and skull base, and mucocele-like lesions resulting from obstruction can grow and could be mistaken for "growing FD" or malignant transformation. Common differential diagnoses of FD include Paget disease, intraosseous meningioma, cemento-ossifying fibroma, chronic osteomyelitis, osteosarcoma, and metastatic disease.

In our reported case, imaging demonstrated an atypical manifestation of FD, featuring an osteolytic lesion with inhomogeneous contrast uptake, which suggested the differential diagnosis lymphoma, metastasis and fibrosarcoma. Since a malignant entity of the tumor could not be excluded by imaging, this resulted in the request for further histological clarification.

\section{Conclusion}


Although FD is a rare disease, radiologists should be aware of this entity and its varying characteristics and consider it as differential diagnosis, in particular in cases of unclear imaging and unusual localizations.

\section{Disclosure}

There are no grants associated with this manuscript. This work or portions of this work have not been previously presented.

\section{Conflict of Interest}

On behalf of all authors, the corresponding author states that there is no conflict of interest.

\section{Acknowledgements}

We thank XYZ for providing the MRI images. 


\section{Figure captions}

Fig 1 High Resolution non-contrast CT of the skull on mastoid level. The arrows demonstrate an osteolytic lesion in the left temporal bone (a), (b, zoomed in) with decent central calcifications (c).

Fig 2 Contrast enhanced MRI images of the skull on the mastoid level. T2-weighted images (a), (b, zoomed in) demonstrate the lesion (arrows) with a low to intermediate signal. Mucus retention within parts of the lesion (c). After intravenous gadolinium application, a slightly inhomogeneous but strong enhancement is visible in the T1-weighted image (d).

Fig 3 Intraoperative photography demonstrating the lesion located in the left mastoid bone.

Fig 4 Histopathology (hematoxylin and eosin stain): Irregular shaped trabeculae of osteoid and woven bone diffusely embedded in a cellular fibrous tissue stroma with minimal or no osteoblast rimming.

Fig 5 Imaging example of a different case with the typical fibrous dysplasia appearance regarding age (adolescent), lesion location (sphenoid bone), and CT imaging characteristics (expansile lesion with a homogenous ground-glass appearance). 


\section{References}

1. Hofmann E, Prescher A. The clivus: anatomy, normal variants and imaging pathology. Clin Neuroradiol. 2012;22(2):123-39. doi:10.1007/s00062-011-0083-4.

2. Kim YH, Song JJ, Choi HG, Lee JH, Oh SH, Chang SO et al. Role of surgical management in temporal bone fibrous dysplasia. Acta Otolaryngol. 2009;129(12):1374-9.

doi:10.3109/00016480902806112.

3. Savu AM MC, Sarafoleanu C. Diagnostic difficulties in fibrous dysplasia - a 5-case series and a literature review. Romanian Journal of Rhinology, . 2014; Vol. 4,(No. 16).

4. Nair SN, Kini R, Rao PK, Bhandarkar GP, Kashyp RR, Rai M et al. Fibrous Dysplasia versus Juvenile Ossifying Fibroma: A Dilemma. Case Rep Dent. 2016;2016:6439026.

doi:10.1155/2016/6439026.

5. Kransdorf MJ, Moser RP, Jr., Gilkey FW. Fibrous dysplasia. Radiographics. 1990;10(3):51937. doi:10.1148/radiographics.10.3.2188311.

6. Leschka SC, Arndt S, Meckel S. [Polyostotic Fibrous dysplasia of the clivus: atypical CT and MRI imaging]. Rofo. 2013;185(1):77-9. doi:10.1055/s-0032-1325341.

7. Chong VF, Khoo JB, Fan YF. Fibrous dysplasia involving the base of the skull. AJR Am J Roentgenol. 2002;178(3):717-20. doi:10.2214/ajr.178.3.1780717.

8. Lustig LR, Holliday MJ, McCarthy EF, Nager GT. Fibrous dysplasia involving the skull base and temporal bone. Arch Otolaryngol Head Neck Surg. 2001;127(10):1239-47.

9. Mahajan S, Kamboj M, Baoz K. Maxillofacial fibrous dysplasia. Indian Journal of Dental Research. 2005;16(1 October 2005):151-2.

10. Towson CE. Monostotic fibrous dysplasia of the mastoid and the temporal bone. AMA Arch Otolaryngol. 1950;52(5):709-24.

11. Putman M. WK, Rahbar R., Gordon C. Fibrous Dysplasia. Pediatric Head and Neck Tumors. 1 ed: Springer-Verlag New York; 2014. p. pp 139-47.

12. Fitzpatrick KA, Taljanovic MS, Speer DP, Graham AR, Jacobson JA, Barnes GR et al. Imaging findings of fibrous dysplasia with histopathologic and intraoperative correlation. AJR Am J Roentgenol. 2004;182(6):1389-98. doi:10.2214/ajr.182.6.1821389. 